\section{ORIGINAL RESEARCH}

\author{
S.-H. Im \\ M.H. Han \\ O.-K. Kwon \\ B.J. Kwon \\ S.H. Kim \\ J.E. Kim \\ C.W. Oh
}

\title{
Endovascular Coil Embolization of 435 Small Asymptomatic Unruptured Intracranial Aneurysms: Procedural Morbidity and Patient Outcome
}

\begin{abstract}
BACKGROUND AND PURPOSE: Whether treatment of small asymptomatic aneurysms is appropriate or not remains controversial. We performed a retrospective study on the procedural morbidity and mortality of coil embolization of small asymptomatic unruptured intracranial aneurysms (UIAs) to obtain a more generalized estimate of procedural risk.
\end{abstract}

MATERIALS AND METHODS: A total of 435 small (maximum diameter $\leq 7 \mathrm{~mm}$ ) asymptomatic UIAs in 370 patients were treated by coil embolization. Aneurysm sizes were determined by using 3D angiograms. We assessed procedure-related morbidity and mortality, immediate postprocedural angiographic results, short-term imaging follow-up results, and clinical outcomes.

RESULTS: Initial aneurysm occlusion was complete in 334 aneurysms, near complete in 78, and incomplete in 22. One internal carotid artery (ICA) aneurysm that ruptured during the procedure was treated with parent artery occlusion. Two hundred wide-neck aneurysms were coiled with the aid of various neck-remodeling techniques. The 44 procedure-related complications were the following: 24 thromboembolisms, 11 coil protrusions or prolapses into the parent vessel, 4 intraprocedural ruptures, 3 device-related complications, and 2 femoral-access complications. We had a total of $44(10.1 \%)$ procedure-related complications with only 1 leading to persistent neurologic deficit. Procedure-related permanent morbidity and mortality were $0.27 \%(1 / 370)$ and $0 \%$, respectively.

CONCLUSIONS: In this series of small unruptured asymptomatic aneurysms, endovascular treatment was achieved with good short-term angiographic outcome and low permanent neurologic impairment. The goal of this study was not to provide a conclusion about treatment guidelines for small UIA but rather to help guide future recommendations by presenting a more generalized estimate of endovascular treatment risk than is currently available.

ncidental aneurysms are now more frequently diagnosed with increased sensitivity and greater availability of neuroimaging techniques. ${ }^{1-4}$ Current evidence in the literature does not conclusively support a standard treatment strategy regarding asymptomatic unruptured intracranial aneurysms (UIAs), especially for small-sized asymptomatic UIAs in patients without a previous subarachnoid hemorrhage (SAH). ${ }^{2}$ Management decisions require an accurate assessment of the risks of various treatment options compared with the natural history of an asymptomatic UIA.

Although we preemptively treat patients with UIAs to avoid the horrendous risks of morbidity and mortality associated with $\mathrm{SAH}$, we often face the decision as to whether to treat a small-sized asymptomatic UIA in a patient without previous SAH. Although accumulating evidence points to the influence of aneurysm size on rupture risk in patients with UIA, the

Received April 2, 2008; accepted after revision July 13.

From the Department of Neurosurgery (S.-H.I.), Dongguk University Hospital, Gyeonggido, Korea; Departments of Radiology (M.H.H., B.J.K., S.H.K.) and Neurosurgery (M.H.H., O.-K.K., J.E.K., C.W.O.), Seoul National University College of Medicine, Seoul, Korea; Clinical Research Institute (M.H.H.), Seoul National University Hospital, Seoul, Korea; and Departments of Radiology (S.H.K.) and Neurosurgery (O.-K.K., C.W.O.), Seoul National University Bundang Hospital, Gyeonggido, Korea.

This work was supported by a grant from the Korea Health 21 R\&D Project, Ministry of Health \& Welfare, Republic of Korea (Grant No: A06-0171-B51004-06N1-00040B).

Please address correspondence to Moon Hee Han, MD, Department of Radiology, Seoul National University Hospital, 28 Yongon-Dong, Chongno-Ku, Seoul 110-744, Korea; e-mail: hanmh@snuh.org

Indicates open access to non-subscribers at www.ajnr.org

DOI 10.3174/ajnr.A1290 critical size at which an aneurysm becomes hazardous remains uncertain..$^{5-7}$ The risk of rupture may be relatively low for small aneurysms, but, to our knowledge, there is no evidence available that confirms their benign natural course or the merits of their conservative management. Estimates on the risk of UIA vary considerably, depending on study design, study population, and aneurysm characteristics. ${ }^{1,2,6}$

To our knowledge, it is not known how many patients with small asymptomatic UIAs have been treated, and no largescale clinical trial devoted to the endovascular treatment of small asymptomatic UIAs has been reported. In the present study, we conducted a retrospective analysis on a group of patients with small $(\leq 7 \mathrm{~mm})$ unruptured UIAs that were treated by endovascular coil embolization, to assess the safety and effectiveness of the procedure and to present a more generalized estimate of endovascular treatment risk than is currently available.

\section{Materials and Methods}

\section{Patient Characteristics}

From May 2002 to December 2006, 1424 aneurysms were occluded with detachable coils at 2 institutions. Of these 1424 aneurysms, 698 had ruptured and 726 had not, and of the 726 unruptured aneurysms, 435 electively coiled small ( $\leq 7 \mathrm{~mm}$ ) asymptomatic aneurysms in 370 patients with no prior SAH were considered the subjects of the present study. There were 276 females $(74.6 \%)$ and 94 males $(25.4 \%)$ with a mean age of 58.0 years (median, 59 years; range, $10-83$ years) (Table 1). The indications for endovascular coiling included surgical difficulty, refusal of surgery, elderly patients, and poor medical condition. An analysis of aneurysm size was performed on the basis of 


\begin{tabular}{lc}
\hline \multicolumn{2}{l}{ Table 1: Characteristics of the patient population } \\
\hline Characteristics & No. of Patients \\
\hline Age (years) & $19(5.14 \%)$ \\
$<40$ & $182(49.2 \%)$ \\
$40-60$ & $169(45.7 \%)$ \\
$>60$ & 58.0 \\
Mean (years) & $10-83$ \\
Range (years) & \\
Sex & $276(74.6 \%)$ \\
Female & $94(25.4 \%)$ \\
Male & \\
\hline
\end{tabular}

precise measurements made on 3D angiograms. All 435 aneurysms were $\leq 7 \mathrm{~mm}$ in maximum diameter. Unruptured aneurysms that presented with symptoms of mass effect or any other aneurysm-related symptoms were excluded. Unruptured aneurysms that were additional to a ruptured aneurysm were excluded. These 435 aneurysms were incidentally discovered during imaging studies performed for clinical reasons unrelated to the presence of an aneurysm. We considered only the primary procedures; secondary procedures performed because of aneurysm recurrence as demonstrated by follow-up angiography were not included in this review.

Procedure-related complications, immediate and follow-up clinical results, and recent patient clinical outcomes were assessed by using patient medical records and by telephone interview with patients or family members. For patients who were not followed up at our institution, clinical outcome was determined by telephone interview. Each patient outcome was graded according to a modified Rankin Scale (mRS). ${ }^{8}$

Complications were defined as neurologic deterioration, abnormal neuroimaging findings, and undesirable events related to the procedures. Morbidity was defined as a change in mRS score associated with the endovascular procedures. ${ }^{8}$

\section{Coiling Procedures}

Coiling of aneurysms was performed on a commercially available biplane angiographic unit (Integris Allura; Philips Medical Systems, Best, the Netherlands) equipped with an image-intensifier matrix of $1024 \times 1024$. Rotational angiography, followed by 3D image reconstruction by volume-rendering, was performed by using the Integris 3D-RA release 3.2 software package (Philips Medical Systems) before the embolization procedure in all cases. On the basis of the images generated with rotational acquisition, at least 2 working projections that provided the best achievable view of the aneurysm neck were defined. At the end of each procedure, postprocedural angiograms in frontal, lateral, and working projections were acquired to rule out any parent artery or branch occlusion.

Four hundred seven and 28 aneurysms were coiled with patients under general or local anesthesia, respectively. Systemic heparinization was performed after placement of the arterial introducer sheath, according to our embolization protocol; 3000 IU of heparin was administered as an intravenous bolus injection, followed by an additional 1000 IU per hour. Heparin was discontinued after embolization in most patients.

All aneurysm embolizations were performed by using detachable coils. The aim of coiling was to pack the aneurysm as densely as possible without compromising the parent artery lumen. Aneurysms were occluded with Guglielmi detachable coils (Boston Scientific, Fremont, Calif) and other bare platinum coils (MicroPlex; MicroVention, Aliso Viejo, Calif; Trufill-DCS, Cordis, Miami Lakes, Fla;

\begin{tabular}{lc}
\hline \multicolumn{2}{l}{ Table 2: Supporting techniques for aneurysm coiling } \\
\hline Adjuvant Technique & No. of Aneurysms \\
\hline Total & $200(46.0 \%, 200 / 435)$ \\
Balloon remodeling & $109(25.1 \%, 109 / 435)$ \\
Stent-assisted & $19(4.37 \%, 19 / 435)$ \\
Multiple-catheter & $77(17.7 \%, 77 / 435)$ \\
2-Catheter & $67(15.4 \%, 67 / 435)$ \\
3-Catheter & $8(1.84 \%, 8 / 435)$ \\
Microcatheter-assisted & $2(0.46 \%, 2 / 435)$ \\
Combination (balloon remodeling, & $5(1.15 \%, 5 / 435)$ \\
2-catheter) & \\
\hline
\end{tabular}

Detach, Cook, Bloomington, Ind). Polymer-coated coils (Matrix, Boston Scientific; HydroCoil, MicroVention) were used in selected cases.

\section{Supporting Devices and Techniques}

Two hundred $(46.0 \%, 200 / 435)$ wide-neck aneurysms were coiled by using various neck-remodeling techniques; there were 109 (25.1\%, 109/435) balloon-remodeling cases, $19(4.37 \%, 19 / 435)$ stent-assisted cases, and 77 (17.7\%, 77/435) multiple-catheter-technique cases (Table 2).

One hundred nine $(25.1 \%)$ wide-neck aneurysms were treated by using the balloon remodeling technique by with an over-thewire balloon microcatheter (Sentry, Boston Scientific; HyperForm or HyperGlide, ev3, Irvine, Calif). Nineteen (4.37\%) wide-neck aneurysms were coiled after placing a permanent supporting device (Neuroform; Boston Scientific). Sixty-seven (15.4\%, 67/435) aneurysms were treated by using a 2 -microcatheter technique. This technique was used in combination with a balloon-assisted technique to treat 5 wide-neck aneurysms. The 3-microcatheter technique was used to treat 8 wide-necked aneurysms, and a microcatheter-assisted technique was used in 2 patients.

\section{Angiographic Results and Follow-Up}

Initial angiographic results of coiling were classified as complete, near complete, or incomplete occlusion. Aneurysm occlusion at the end of the procedure was considered to be complete when the aneurysmal sac and neck were packed and no contrast filling of the aneurysm sac was confirmed by angiography. Occlusion was considered near complete when the sac was occluded but a neck remnant was present and as incomplete when there was persistent contrast filling of the aneurysmal sac remnant.

Patients were scheduled for a follow-up visit and a plain radiographic examination in an outpatient clinic usually between 3 and 6 months after coiling. Our normal further follow-up protocol is to perform plain radiography and MR angiography (MRA) at 6 months and conventional angiography at 1 year after coiling. Seventy-one patients underwent follow-up conventional angiography, and 282 patients underwent follow-up MRA. Follow-up plain radiographic examinations consisted of acquiring frontal, lateral, and working projections, which were determined according to the locations of patients' bony landmarks as they had been defined during initial endovascular coiling. Neurologic status was evaluated during every visit.

An aneurysm was considered to be recurrent if a previously completely occluded aneurysm was found to have partial or even less recanalization of the neck or sac. 


\begin{tabular}{|c|c|c|}
\hline Location & $\begin{array}{l}\text { No. of Aneurysms } \\
(\%)\end{array}$ & $\begin{array}{c}\text { Aneurysm Size } \\
\text { (mean) }\end{array}$ \\
\hline Anterior circulation & $390(89.7)$ & 4.52 \\
\hline ICA-ophthalmic & $10(2.3)$ & 4.76 \\
\hline ICA bifurcation & $12(2.3)$ & 4.52 \\
\hline Cavernous ICA & $16(3.68)$ & 4.89 \\
\hline ICA-anterior choroidal artery & $18(4.14)$ & 4.39 \\
\hline $\begin{array}{l}\text { ICA-superior hypophyseal } \\
\text { artery }\end{array}$ & $55(12.6)$ & 3.93 \\
\hline ICA-other locations & $120(27.6)$ & 4.47 \\
\hline ACA & $18(4.14)$ & 4.81 \\
\hline Posterior communicating artery & 38 (8.74) & 4.53 \\
\hline MCA & $46(10.6)$ & 4.37 \\
\hline Anterior communicating artery & $57(13.1)$ & 4.88 \\
\hline Posterior circulation & $45(10.3)$ & 4.22 \\
\hline $\begin{array}{l}\text { Anterior inferior cerebellar } \\
\text { artery }\end{array}$ & $2(0.46)$ & 3.67 \\
\hline $\begin{array}{l}\text { Posterior inferior cerebellar } \\
\text { artery }\end{array}$ & $2(0.46)$ & 4.19 \\
\hline Basilar trunk & $2(0.46)$ & 2.22 \\
\hline Vertebral artery & $4(0.92)$ & 5.81 \\
\hline Posterior cerebral artery & $5(1.15)$ & 3.37 \\
\hline Superior cerebellar artery & $10(2.3)$ & 3.40 \\
\hline Basilar tip & $20(4.60)$ & 4.78 \\
\hline
\end{tabular}

Note:-ICA indicates internal carotid artery; ACA, anterior cerebral artery; MCA, middle cerebral artery.

\section{Results}

\section{Locations and Sizes of Aneurysms}

The most frequent aneurysm location was the internal carotid artery (ICA) $(n=231)$, followed by the anterior communicating artery $(n=57)$, the middle cerebral artery (MCA) $(n=$ $46)$, the posterior circulation $(n=45)$, and the posterior communicating artery $(n=38)$ (Table 3$)$. Three hundred ninety $(89.7 \%, 390 / 435)$ aneurysms were located in the anterior circulation (ie, anterior communicating artery, 57; MCA, 46; posterior communicating artery, 38; anterior cerebral artery [ACA], 18; ICA-superior hypophyseal artery, 55; ICA-anterior choroidal artery, 18; cavernous ICA, 16; ICA bifurcation, 12; ICA-ophthalmic artery, 10; and ICA-other locations, 120). Forty-five of the $435(10.3 \%, 45 / 435)$ aneurysms were located in the posterior circulation (ie, basilar tip, 20; superior cerebellar artery, 10; posterior cerebral artery, 5; vertebral artery, 4; basilar trunk, 2; posterior inferior cerebellar artery, 2; and anterior inferior cerebellar artery, 2).

Aneurysmal sac sizes ranged from 2.0 to $7.0 \mathrm{~mm}$ in maximum diameter. The mean size of the 435 unruptured aneurysms was $4.49 \mathrm{~mm}$ (median, $4.72 \mathrm{~mm}$ ). Aneurysm sac sizes were $<3 \mathrm{~mm}$ for 47 aneurysms, $3-5 \mathrm{~mm}$ for 242 , and $>5 \mathrm{~mm}$ for 146 .

\section{Aneurysm Outcome}

At the end of the procedure, aneurysm occlusion was complete for $334(76.8 \%)$ of the 435 aneurysms, near complete for 78 (17.9\%) aneurysms, and incomplete for 22 (5.06\%) aneurysms. One ICA aneurysm that ruptured during the procedure was treated with parent artery occlusion. No further treatment was planned for the 22 incompletely occluded aneurysms because sac remnants were not large enough to warrant retreatment. Follow-up imaging evaluations with plain radiography, MRA, and/or conventional angiography were performed on
358 aneurysms in 306 patients for 6 months or more after embolization.

Three hundred fifty-eight $(82.3 \%)$ of the 435 aneurysms were examined during follow-up by plain radiography and/or MRA and/or conventional angiography for $\geq 6$ months after embolization. These aneurysms were followed up for a cumulative period of 5064 months (mean, 14.2 months). Sustained stable aneurysm occlusion was achieved for 337 aneurysms (94.1\%). Twenty-one (5.87\%) of the 358 aneurysms that were followed up recurred. Of these 21 lesions, 15 with small neck recurrences were not candidates for a second treatment because these recurrences appeared to be too small for further coil placement. The other 6 aneurysms required repeat coil embolization. The reason for repeat embolization was asymptomatic aneurysmal recanalization due to coil compaction without an increase in aneurysm size in 5 cases. The remaining one was an anterior communicating artery aneurysm, which was observed to have increased in size with coil loosening by follow-up conventional angiography 7 months after embolization. Time lapses between first and second embolizations ranged from 1 month to 28 months (mean, 13.3 months). Complete or near-complete aneurysm occlusion was achieved in 5 retreated patients without procedure-related morbidity or mortality. One recurrent aneurysm (an anterior communicating artery aneurysm with a wide neck) was surgically clipped after unsuccessful repeat coil embolization.

One patient experienced bleeding 18 months after complete coil embolization of a 5-mm MCA bifurcation aneurysm. He had been lost to clinical follow-up after initial coil embolization. This ruptured aneurysm was completely occluded by the second coil embolization. The patient had a good recovery without neurologic deficits.

Clinical follow-up was available in all 370 patients. Clinical follow-up periods varied from 6 months to 5 years and averaged 21.5 months. One patient died of a car crash, and another patient died because of a ruptured untreated aneurysm. The 1 patient with permanent morbidity was independent (mRS score, 2) at last follow-up, 4 years after the procedure. The other 367 patients resumed their previous daily activities.

\section{Treatment-Related Complications}

Forty-four $(10.1 \%, 44 / 435)$ procedure-related complications occurred. Complications included 24 thromboembolisms (5.52\%), 4 intraprocedural ruptures $(0.92 \%), 3$ device-related complications $(0.69 \%), 11$ coil protrusions or prolapses into the parent vessel $(2.53 \%)$, and 2 femoral access complications $(0.46 \%)$ (Table 4$)$. Of these, 43 complications had no persistent neurologic consequence, but 1 resulted in a persistent neurologic deficit ( $\mathrm{mRS}$ score of 2 at last follow-up). The overall rate of treatment-related morbidity was $1.15 \%$ (5/435) (ie, 3 cases of neurologic deficits and 2 cases of chemical meningitis). Procedure-related permanent morbidity and mortality rates were $0.27 \%$ and $0 \%$, respectively.

Thromboembolic complications occurred during 24 of the 435 procedures, and 1 of these resulted in a persistent neurologic deficit (ie, right hemiparesis), which was in keeping with the consequences of a left cerebral infarct secondary to MCA branch occlusion. Two resulted in transient neurologic deficits that resolved within 24 hours postoperatively. Twenty-one thromboembolic complications were asymptomatic (ie, 18 


\begin{tabular}{lc}
\hline Table 4: Procedure-related complications & \\
\hline Complications & No. of Cases \\
\hline Total & $44(10.1 \%, 44 / 435)$ \\
Thromboembolism & $24(5.52 \%, 24 / 435)$ \\
Asymptomatic & $21(4.83 \%, 21 / 435)$ \\
Symptomatic & $3(0.69 \%, 3 / 435)$ \\
Transient neurologic deficit & $2(0.46 \%, 2 / 435)$ \\
Permanent neurologic deficit & $1(0.23 \%, 1 / 435)$ \\
Coil protrusion or prolapse & $11(2.53 \%, 11 / 435)$ \\
No flow compromise & $8(1.84 \%, 8 / 435)$ \\
Flow compromise & $3(0.69 \%, 3 / 435)$ \\
Intraprocedural ruptures & $4(0.92 \%, 4 / 435)$ \\
Slow leak & $3(0.69 \%, 3 / 435)$ \\
Massive hemorrhage & $1(0.23 \%, 1 / 435)$ \\
Device-related & $3(0.69 \%, 3 / 435)$ \\
HydroCoil-associated chemical & $2(0.46 \%, 2 / 435)$ \\
meningitis & \\
Coil fracture & $1(0.23 \%, 1 / 435)$ \\
Femoral access complications & $2(0.46 \%, 2 / 435)$ \\
Femoral arteriovenous fistula & $1(0.23 \%, 1 / 435)$ \\
External iliac artery dissection & $1(0.23 \%, 1 / 435)$ \\
\hline
\end{tabular}

small thrombi in parent arteries or small branches without flow compromise and 3 small branch occlusions). Of the 24 thromboembolic complications, $9(37.5 \%, 9 / 24)$ occurred in patients with wide-neck aneurysms treated with remodeling techniques. In the 24 cases with thromboembolic events, aneurysm locations were the anterior communicating artery in 6 patients, the ICA in 9, the MCA in 3, the posterior communicating artery in 4 , the basilar top in 1, and the distal ACA in 1 patient. All thromboembolic complications were diagnosed during the procedure. In the patients who had thromboembolic complications, an intravenous anticoagulation with heparin was sustained for 24 hours. In 1 patient with thromboembolic occlusion of an MCA branch, intra-arterial thrombolysis by using abciximab (ReoPro) was performed; however, recanalization was unsuccessful and the patient had a persistent neurologic deficit consistent with a left MCA territory infarct. Brain MR imaging was performed in patients with symptomatic thromboembolic complications. Four thromboembolic complications were associated with device-assisted embolization (ie, 2 Neuroform-assisted embolizations and 2 balloonassisted embolizations). Five thromboembolic complications were associated with the 2-microcatheter technique.

Device-related complications included 1 coil fracture (the fractured coil was retrieved by using a snare retriever) and 2 HydroCoil-associated cases of chemical meningitis. There were 8 coil protrusions into the parent artery with no flow compromise, and 3 coil prolapses into the parent vessel with flow compromise (protruded coil loops were stabilized with a stent at the parent vessel wall).

Four aneurysms perforated during procedures with protrusion of coils outside the aneurysms (Table 5). Leakage of contrast media was demonstrated in 3 of the 4 aneurysms during aneurysm filling, and further aneurysmal packing continued. In these 3 patients, CT after embolization showed SAH, but all patients recovered well without neurologic deficit. One 2-mm aneurysm on the right paraclinoid ICA, which ruptured during the procedure, was treated by parent-artery occlusion with a detachable balloon and coils after angiographically confirming collateral flow through the anterior communicating

\begin{tabular}{lccc}
\hline \multicolumn{3}{c}{ Table 5: Clinical findings of patients with an intraoperative rupture } \\
\hline Case & $\begin{array}{c}\text { Age } \\
\text { (years) }\end{array}$ & Aneurysm Location & $\begin{array}{c}\text { Aneurysm Size } \\
(\mathrm{mm})\end{array}$ \\
\hline 1 & 31 & ICA-superior hypophyseal & 2.32 \\
2 & 58 & MCA-superior division & 5.54 \\
3 & 70 & MCA-bifurcation & 4.10 \\
4 & 79 & Anterior communicating artery & 6.21 \\
\hline
\end{tabular}

artery due to the failure of aneurysm coiling. The patient recovered without any neurologic deficit.

\section{Femoral-Access Complications Included 1 Femoral Arteriovenous Fistula and 1 External Iliac Artery Dissection}

The length of stay in the hospital was dependent on the condition of the patients after the procedures. In patients without complications and in patients with minor complications such as asymptomatic thromboembolism, they typically had a hospital stay of 2 or 3 days. In patients with significant complications such as symptomatic thromboembolism and meningitis, hospital stay extended several weeks.

\section{Discussion}

Increased sensitivity and the greater availability of neuroimaging techniques have resulted in the frequent detection of asymptomatic UIAs, and because aneurysmal SAH frequently causes devastating neurologic injury, prevention has been advocated as the most effective strategy with the intention of reducing mortality rates. Although the risk of rupture of asymptomatic UIAs and treatment morbidity and mortality have been extensively investigated, current evidence does not conclusively support a standard treatment strategy regarding asymptomatic UIAs; and in particular, the size at which an aneurysm becomes dangerous remains uncertain. ${ }^{2,7}$ All current treatments carry risks, and recommendations for treatment versus observation are often difficult and controversial. Management decisions require accurate assessments of the risks of potential rupture, death, or disability due to rupture and the risks related to various treatment options to prevent an iatrogenic insult to patients destined to coexist peacefully with their unruptured aneurysms before dying of some other cause.

There is considerable disagreement concerning the management of small-sized asymptomatic UIAs, even with respect to the relative rupture risk presented by these aneurysms. ${ }^{6,9}$ Moreover, current understanding does support the use of the small aneurysmal diameter as an independent criterion for management decision-making, though it is of undoubted importance..$^{5-7}$ The annual bleeding rate of asymptomatic UIAs has been investigated by retrospective and prospective studies. ${ }^{2,3,5,10-21}$ However, the estimates of UIA rupture risk vary considerably and appear to depend on study design, study population, and aneurysm characteristics. ${ }^{1,2,6}$ In a study by Wiebers et al, ${ }^{6} 5$-year cumulative rupture rates for UIAs located in the ICA, anterior communicating artery, ACA, or MCA were $0 \%, 2.6 \%, 14.5 \%$, and $40 \%$ for aneurysms $<7 \mathrm{~mm}$, 7-12 mm, 13-24 mm, and $\geq 25 \mathrm{~mm}$, respectively, compared with rates of $2.5 \%, 14.5 \%, 18.4 \%$, and $50 \%$, respectively, for the same-sized categories involving posterior circulation and 
posterior communicating artery aneurysms. In other studies, the annual ranges of rupture rates among patients with asymptomatic UIA varied from $0.05 \%$ to $2.3 \%$ per year. ${ }^{7,21,22}$

In 1998, the International Study of Unruptured Intracranial Aneurysms (INSUIA) investigators reported the results of a large retrospective multicenter study on the natural course of asymptomatic UIAs. ${ }^{5}$ In this study, the natural course of 1937 asymptomatic UIAs in 1449 patients was retrospectively assessed. The findings obtained suggested that annual hemorrhage rates of aneurysms $<10 \mathrm{~mm}, 10-25 \mathrm{~mm}$, and $>25 \mathrm{~mm}$ in diameter in patients with no history of SAH were $0.05 \%$, approximately $1 \%$, and $6 \%$, respectively. Moreover, this study suggested that small UIAs have a benign natural course and a very low risk of bleeding when left untreated. ${ }^{5}$ After this seminal publication, many cerebrovascular centers moved away from treating small incidentally discovered aneurysms, particularly in older patients. However, discrepancies exist between the ISUIA and other studies, and serious flaws were identified in the ISUIA study concerning its retrospective nature, the short follow-up period, and recruitment bias. ${ }^{6,7,9}$ Ideally, the natural history of UIAs should be determined by a prospective study with no treatment and by long-term follow-up, but it is unrealistic to expect that such a study will ever be conducted. Thus, all natural history studies have been performed in patients selected for conservative management, which likely influences results.

Although the risk of rupture may be relatively low for aneurysms, no confirmatory evidence validates the use of conservative management for the treatment of small asymptomatic UIAs., ${ }^{2,6}$ Many authors recommend treatment for asymptomatic aneurysms incidentally discovered because these lesions present a substantial rupture risk. ${ }^{10,11,22,23}$

Preventive endovascular coil embolization of unruptured aneurysms requires reliable data on its risks and benefits. Preventive treatment of unruptured aneurysms should be undertaken by experienced neurointerventionists to obtain the best possible outcomes. Moreover, technologic innovations and optimized treatment regimens, such as antiplatelet and anticoagulation, have significantly reduced morbidity rates in recent years. ${ }^{24}$ Our series, in which 370 patients were treated with endovascular coils for small asymptomatic UIAs, found a low permanent complication rate of $0.27 \%$. In our series, elective coiling of small UIAs was associated with low procedural morbidity $(1.15 \%)$ and mortality $(0 \%)$, despite a high proportion of technically challenging procedures. However, our follow-up was short; therefore, the true permanent complications are really not known. Moreover, the true significance of our low procedural morbidity and mortality may not be known because we do not know how many of the thromboembolic complications were associated with abnormalities on diffusion-weighted MR imaging, and subtle cognitive and neurologic abnormalities might be missed in cases with thromboembolic complications.

Growing evidence indicates that endovascular coiling carries lower risks than surgical clipping for unruptured aneurysms: In a direct comparison of surgical and endovascular repair of unruptured aneurysms in 130 patients, Johnston et $\mathrm{al}^{25,26}$ found that $25 \%$ of patients in the surgical group developed a change in mRS score of $\geq 2$ versus only $8 \%$ of endovascular patients. Although direct comparisons may be invalid because of differences in patient and aneurysm characteristics, procedural morbidity found in the present study is superior or similar to that of surgically treated unruptured aneurysms. For example, in a meta-analysis of 733 patients in 28 surgical series, mortality and morbidity were $1.0 \%$ and $4.1 \%$, respectively, ${ }^{27}$ and in another meta-analysis conducted by Raaymakers et al, ${ }^{28}$ morbidity and mortality were $10.9 \%$ and $2.6 \%$ for surgically treated unruptured aneurysm in 2460 patients. A prospective study of 202 patients found that morbidity and mortality totaled $1.0 \%$ for aneurysms $<10 \mathrm{~mm}, 5.0 \%$ for those between 11 and $25 \mathrm{~mm}$, and $20 \%$ for those $>25 \mathrm{~mm} .{ }^{29}$ Our results fall in the same range as those in previous reports on endovascular treatment. In a systematic review of 30 studies comprising 1397 unruptured aneurysms treated with detachable coils, morbidity and mortality were $7 \%$ and $0.6 \%$, respectively ${ }^{24}$; and in a systematic review of 176 unruptured aneurysms in 149 patients treated with detachable coils, morbidity and mortality were $2.6 \%$ and $1.3 \%$, respectively. ${ }^{30}$ However, the estimates of clipping risk vary considerably and appear to depend on various factors such as study population and aneurysm characteristics. In a study by Ogilvy and Carter, ${ }^{31}$ the true morbidity with clipping simple small anterior circulation aneurysms was quite small. Clinicians should also be reminded that the long-term efficacy and durability of endovascular treatment for unruptured aneurysms remains to be determined.

Although endovascular coil embolization appears to be used with increasing frequency, it requires long-term follow-up to ensure the absence of recurrence. ${ }^{26}$ Because UIA embolization is performed to prevent rupture, bleeding rate is considered a measure of the effectiveness of the procedure. Ideally, the effectiveness of coil embolization of asymptomatic UIAs should be evaluated by comparing their natural courses with postembolization bleeding rates. However, to our knowledge, the natural course of asymptomatic UIAs and the longterm bleeding rates of embolized aneurysms have not been precisely determined. Moreover, because few large trials have been conducted to study the endovascular treatment outcomes of small asymptomatic UIAs, it is unclear whether coil embolization actually reduces the incidence of bleeding. Because of an inadequate follow-up period, our series does not have the statistical power to prove the effectiveness of coiling or to determine the risk of bleeding of embolized small UIAs of $\leq 7 \mathrm{~mm}$. The long-term efficacy in terms of preventing hemorrhage from coiling a $\leq 3-\mathrm{mm}$ aneurysm is also not known. It is also uncertain how incomplete coil embolization affects UIA bleeding rates because our follow-up was short. Our series suggests that endovascular treatment of UIAs does not provide absolute protection, though bleeding after coil embolization was a rare event. However, we believe that the findings of the present study are worth considering when making treatment decisions in patients with small UIAs and may provide partial scientific validation to justify an embolization for a small asymptomatic UIA. Longer clinical and neuroimaging follow-up periods are necessary to determine more accurately the effect of endovascular treatment in small unruptured UIAs.

In our series, 2 of 44 procedure-related complications occurred in 2-mm aneurysms. In 1 case, the coil prolapsed into the parent vessel with flow compromise, and protruded coil 
loops were stabilized with a stent at the parent vessel wall. The other aneurysm, which ruptured during the procedure, was treated by parent artery occlusion with detachable balloon and coils. The treatment of very small cerebral aneurysms with maximal diameter $<3 \mathrm{~mm}$ remains a challenge for endovascular and surgical treatment. Endovascular treatment of these lesions may be difficult and associated with a high risk of complications because of their small size. Such lesions might be dangerous enough to warrant therapy as in our 2 patients, though coil embolizations were carried out without difficulty in most 2-mm aneurysms in our series. Our series include 16 cases of cavernous aneurysms that were treated in the early days of our experience, and later we did not perform endovascular treatment of asymptomatic unruptured cavernous aneurysms.

\section{Conclusions}

The potential rupture risk of small asymptomatic unruptured aneurysms and the benefits of prophylactic treatment remain open issues. We assessed the procedural safety of the endovascular coiling of small asymptomatic unruptured aneurysms and found that endovascular treatment of small UIAs was performed successfully without inducing neurologic deficits in most patients.

The goal of this study was not to provide a conclusion about treatment guidelines for small UIAs but rather to help guide future recommendations by presenting a more generalized estimate of endovascular treatment risk than is currently available.

\section{References}

1. Inagawa T, Hirano A. Autopsy study of unruptured incidental intracranial aneurysms. Surg Neurol 1990;34:361-65

2. Rinkel GJ, Djibuti M, Algra A, et al. Prevalence and risk of rupture of intracranial aneurysms: a systematic review. Stroke 1998;29:251-56

3. Rosenørn J, Eskesen V. Patients with ruptured intracranial saccular aneurysms: clinical features and outcome according to the size. Br J Neurosurg 1994;8:73-78

4. King JT Jr, Glick HA, Mason TJ, et al. Elective surgery for asymptomatic, unruptured, intracranial aneurysms: a cost-effectiveness analysis. J Neurosurg 1995;83:403-12

5. Unruptured intracranial aneurysms: risk of rupture and risks of surgical intervention-International Study of Unruptured Intracranial Aneurysms Investigators. $N$ Engl J Med 1998;339:1725-33. Erratum in: N Engl J Med 1999;340:744

6. Wiebers DO, Whisnant JP, Huston J 3rd, et al, for the International Study of Unruptured Intracranial Aneurysms Investigators. Unruptured intracranial aneurysms: natural history, clinical outcome, and risks of surgical and endovascular treatment. Lancet 2003;362:103-10
7. Zuccarello M. Treatment strategy for patients with unruptured intracranial aneurysms. Neurol Med Chir (Tokyo) 2001;41:571-75

8. Rankin J. Cerebral vascular accidents in patients over the age of 60. II. Prognosis. Scott Med J 1957;2:200-15

9. Weir B. Unruptured intracranial aneurysms: a review. J Neurosurg 2002;96:3-42

10. Dickey P, Nunes J, Bautista C, et al. Intracranial aneurysms: size, risk of rupture, and prophylactic surgical treatment. Conn Med 1994;58:583-86

11. Yasui N, Magarisawa S, Suzuki A, et al. Subarachnoid hemorrhage caused by previously diagnosed, previously unruptured intracranial aneurysms: a retrospective analysis of 25 cases. Neurosurgery 1996;39:1096-100

12. Asari S, Ohmoto T. Long-term outcome of surgically treated unruptured cerebral aneurysms. Clin Neurol Neurosurg 1994;96:230-35

13. Barrow DL, Reisner A. Natural history of intracranial aneurysms and vascular malformations. Clin Neurosurg 1993;40:3-39

14. Inagawa $\mathrm{T}$, Hada $\mathrm{H}$, Katoh $\mathrm{Y}$. Unruptured intracranial aneurysms in elderly patients. Surg Neurol 1992;38:364-70

15. Juvela S, Porras M, Heiskanen O. Natural history of unruptured intracranial aneurysms: a long-term follow-up study. J Neurosurg 1993;79:174-82

16. Mizoi K, Yoshimoto T, Nagamine Y, et al. How to treat incidental cerebral aneurysms: a review of 139 consecutive cases. Surg Neurol 1995;44:114-20

17. Orz Y, Kobayashi S, Osawa M, et al. Aneurysm size: a prognostic factor for rupture. Br J Neurosurg 1997;11:144-49

18. Taylor CL, Yuan Z, Selman WR, et al. Cerebral arterial aneurysm formation and rupture in 20,767 elderly patients: hypertension and other risk factors. J Neurosurg 1995;83:812-19

19. Wiebers DO, Whisnant JP, O'Fallon WM. The natural history of unruptured intracranial aneurysms. N Engl J Med 1981;304:696-98

20. Wiebers DO, Whisnant JP, Sundt TM Jr, et al. The significance of unruptured intracranial saccular aneurysms. J Neurosurg 1987;66:23-29

21. Yasui N, Suzuki A, Nishimura H, et al. Long-term follow-up study of unruptured intracranial aneurysms. Neurosurgery 1997;40:1155-59

22. Juvela S, Porras M, Poussa K. Natural history of unruptured intracranial aneurysms: probability of and risk factors for aneurysm rupture. J Neurosurg 2000;93:379-87

23. Solomon RA, Correll JW. Rupture of a previously documented asymptomatic aneurysm enhances the argument for prophylactic surgical intervention. Surg Neurol 1988;30:321-23

24. Lanterna LA, Tredici G, Dimitrov BD, et al. Treatment of unruptured cerebral aneurysms by embolization with Guglielmi detachable coils: case-fatality, morbidity, and effectiveness in preventing bleeding - a systematic review of the literature. Neurosurgery 2004;55:767-75

25. Johnston SC, Zhao S, Dudley RA, et al. Treatment of unruptured cerebral aneurysms in California. Stroke 2001;32:597-605

26. Johnston SC, Wilson CB, Halbach VV, et al. Endovascular and surgical treatment of unruptured cerebral aneurysms: comparison of risks. Ann Neurol 2000;48:11-19

27. King JT Jr, Berlin JA, Flamm ES. Morbidity and mortality from elective surgery for asymptomatic, unruptured, intracranial aneurysms: a meta-analysis. J Neurosurg 1994;81:837-42

28. Raaymakers TW, Rinkel GJ, Limburg M, et al. Mortality and morbidity of surgery for unruptured intracranial aneurysms: a meta-analysis. Stroke 1998;29:1531-38

29. Solomon RA, Fink ME, Pile-Spellman J. Surgical management of unruptured intracranial aneurysms. J Neurosurg 1994;80:440-46

30. van Rooij WJ, Sluzewski M. Procedural morbidity and mortality of elective coil treatment of unruptured intracranial aneurysms. AJNR Am J Neuroradiol 2006;27:1678-80

31. Ogilvy CS, Carter BS. Stratification of outcome for surgically treated unruptured intracranial aneurysms. Neurosurgery 2003;52:82-87 